\title{
Wavelet entropy of stochastic processes
}

\author{
L. Zunino ${ }^{\mathrm{a}, \mathrm{b}, \mathrm{c}, *}$, D.G. Pérez ${ }^{\mathrm{d}}$, M. Garavaglia ${ }^{\mathrm{a}, \mathrm{c}}$, O.A. Rosso ${ }^{\mathrm{e}}$ \\ ${ }^{a}$ Centro de Investigaciones Ópticas (CIOP), CC. 124 Correo Central, 1900 La Plata, Argentina \\ ${ }^{\mathrm{b}}$ Departamento de Ciencias Básicas, Facultad de Ingenierí, Universidad Nacional de La Plata (UNLP), 1900 La Plata, Argentina \\ ${ }^{\mathrm{c}}$ Departamento de Fisica, Facultad de Ciencias Exactas, Universidad Nacional de La Plata (UNLP), 1900 La Plata, Argentina \\ ${ }^{\mathrm{d}}$ Instituto de Física, Pontificia Universidad Católica de Valparaíso (PUCV), 23-40025 Valparaíso, Chile \\ ${ }^{\mathrm{e}}$ Facultad de Ciencias Exactas y Naturales, Instituto de Cálculo, Universidad de Buenos Aires (UBA), Pabellón II, Ciudad Universitaria, \\ 1428 Ciudad de Buenos Aires, Argentina
}

Received 10 January 2006; received in revised form 30 October 2006

Available online 28 February 2007

\begin{abstract}
We compare two different definitions for the wavelet entropy associated to stochastic processes. The first one, the normalized total wavelet entropy (NTWS) family [S. Blanco, A. Figliola, R.Q. Quiroga, O.A. Rosso, E. Serrano, Time-frequency analysis of electroencephalogram series, III. Wavelet packets and information cost function, Phys. Rev. E 57 (1998) 932-940; O.A. Rosso, S. Blanco, J. Yordanova, V. Kolev, A. Figliola, M. Schürmann, E. Başar, Wavelet entropy: a new tool for analysis of short duration brain electrical signals, J. Neurosci. Method 105 (2001) 65-75] and a second introduced by Tavares and Lucena [Physica A 357(1) (2005) 71-78]. In order to understand their advantages and disadvantages, exact results obtained for fractional Gaussian noise $(-1<\alpha<1)$ and fractional Brownian motion $(1<\alpha<3)$ are assessed. We find out that the NTWS family performs better as a characterization method for these stochastic processes.
\end{abstract}

(C) 2007 Elsevier B.V. All rights reserved.

Keywords: Wavelet analysis; Wavelet entropy; Fractional Brownian motion; Fractional Gaussian noise; $\alpha$-parameter

\section{Introduction}

The advantages of projecting an arbitrary continuous stochastic process in a discrete wavelet space are widely known. The wavelet time-frequency representation does not make any assumptions about signal stationarity and is capable of detecting dynamic changes due to its localization properties. Unlike the harmonic base functions of the Fourier analysis, which are precisely localized in frequency but infinitely extend in time, wavelets are well localized in both time and frequency. Moreover, the computational time is significantly shorter since the algorithm involves the use of fast wavelet transform in a multi-resolution framework. Finally, contaminating noise contributions can be easily eliminated when they are concentrated in

\footnotetext{
*Corresponding author. Centro de Investigaciones Ópticas (CIOp), CC. 124 Correo Central, 1900 La Plata, Argentina. Tel.: + 542214714341 ; fax: +542214717872 .

E-mail addresses: lucianoz@ciop.unlp.edu.ar (L. Zunino), dario.perez@ucv.cl (D.G. Pérez), garavagliam@ciop.unlp.edu.ar (M. Garavaglia), oarosso@fibertel.com.ar (O.A. Rosso).
} 
some frequency bands [1,2]. These important reasons justify the introduction, within this special space, of entropy-based algorithms in order to quantify the degree of order or disorder associated with a multifrequency signal response. With the entropy estimated via the wavelet transform, the time evolution of frequency patterns can be followed with an optimal time-frequency resolution. Several recent papers have confirmed the effectiveness, relevance and suitability of the wavelet entropy as a quantifier of experimental and synthetic signals. These include applications to the characterization of brain electrical signals (EEG and EP/ ERP) and neuronal activity [3-14]), solar physics [15,16], erythrocytes deformation [17], laser propagation throughout turbulent media and other lasers applications [18-20], pseudo-random number generators [21], the quantum-classical limit [22], and fractional Brownian motion [23].

In this paper we focus on two definitions for this quantifier: the normalized total wavelet entropy (NTWS) family introduced by one of us (O.A. Rosso) [3,4], and another definition given recently by Tavares and Lucena [24]. We compare their performances while characterizing two important stochastic processes: the fractional Brownian motion ( $\mathrm{fBm}$ ) and the fractional Gaussian noise (fGn). They have been employed as stochastic models in different and heterogeneous scientific fields, like atmospheric turbulence [18,19], econophysics [25] and coastal dispersion [26]. We will show that the NTWS family gives a better characterization for both of them.

\section{Wavelet quantifiers}

\subsection{Wavelet energies}

The wavelet analysis is one of the most useful tools when dealing with data samples. Any signal can be decomposed by using a wavelet dyadic discrete family $\left\{2^{j / 2} \psi\left(2^{j} t-k\right)\right\}$, with $j, k \in \mathbb{Z}$ (the set of integers) -an orthonormal basis for $L^{2}(\mathbb{R})$ consisting of finite-energy signals - of translations and scaling functions based on a function $\psi$ : the mother wavelet [1,2]. In the following, given a stochastic process $s(t)$ its associated signal is assumed to be given by the sampled values $\mathscr{S}=\{s(n), n=1, \ldots, M\}$. Its wavelet expansion has associated wavelet coefficients given by

$$
C_{j}(k)=\left\langle\mathscr{S}, 2^{j / 2} \psi\left(2^{j} t-k\right)\right\rangle,
$$

with $j=-N, \ldots,-1$, and $N=\log _{2} M$. The number of coefficients at each resolution level is $N_{j}=2^{j} M$. Note that this correlation gives information on the signal at scale $2^{-j}$ and time $2^{-j} k$. The set of wavelet coefficients at level $j,\left\{C_{j}(k)\right\}_{k}$, is also a stochastic process where $k$ represents the discrete time variable. It provides a direct estimation of local energies at different scales. Inspired by the Fourier analysis we define the energy at resolution level $j$ by

$$
\mathscr{E}_{j}=\sum_{k} \mathbb{E}\left|C_{j}(k)\right|^{2}
$$

where $\mathbb{E}$ stands for the average using some, at first, unknown probability distribution. In the case the set $\left\{C_{j}(k)\right\}_{k}$ is proved to be a stationary process the previous equation reads

$$
\mathscr{E}_{j}=N_{j} \mathbb{E}\left|C_{j}(k)\right|^{2} .
$$

Observe that the energy $\mathscr{E}_{j}$ is only a function of the resolution level. Also, under the same assumptions, the temporal average energy at level $j$ is given by

$$
\widetilde{\mathscr{E}}_{j}=\frac{1}{N_{j}} \sum_{k} \mathbb{E}\left|C_{j}(k)\right|^{2}=\mathbb{E}\left|C_{j}(k)\right|^{2},
$$

where we have used Eq. (3) to arrive to the last step in this equation. Since we are using dyadic discrete wavelets the number of coefficients decreases over the low frequency bands (at resolution level $j$ the number is halved with respect to the previous one $j+1$ ); thus, the latter energy definition reinforces the contribution of these low frequency bands. 
Summing over all the available wavelets levels $j$ we obtain the corresponding total energies: $\mathscr{E}$ total $=$ $\sum_{j=-N}^{-1} \mathscr{E}_{j}$ and $\widetilde{\mathscr{E}}_{\text {total }}=\sum_{j=-N}^{-1} \widetilde{\mathscr{E}}_{j}$. Finally, we define the relative wavelet energy

$$
p_{j}=\frac{\mathscr{E}_{j}}{\mathscr{E}_{\text {tot }}}
$$

and the relative temporal average wavelet energy

$$
\widetilde{p}_{j}=\frac{\widetilde{\mathscr{E}}_{j}}{\widetilde{\mathscr{E}}_{\text {tot }}} .
$$

Clearly, $\sum_{j=-N}^{-1} p_{j}=\sum_{j=-N}^{-1} \widetilde{p}_{j}=1$; both define probability distributions: $\left\{p_{j}\right\}$ and $\left\{\widetilde{p}_{j}\right\}$. They can also be considered as scale energy densities because supply information about the relative energy associated to each frequency band. So, they enable us to learn about their corresponding degree of importance.

\subsection{NTWS family}

The Shannon entropy [27] provides a measure of the information of any distribution. Consequently, we have previously defined the family of NTWS as [3,4]

$$
S_{\mathrm{W}}(N)=-\sum_{j=-N}^{-1} p_{j} \cdot \log _{2} p_{j} / S_{\max }
$$

and

$$
\widetilde{S}_{\mathrm{W}}(N)=-\sum_{j=-N}^{-1} \widetilde{p}_{j} \cdot \log _{2} \widetilde{p}_{j} / S_{\max },
$$

with $S_{\max }=\log _{2} N$. It has been adopted the base-2 logarithm for the entropy definition to take advantage of the dyadic nature of the wavelet expansion; thus, simplifying the entropy formulae that will be used in this work. To estimate these quantifiers two different strategies have been adopted: the average and mean NTWS - further details about these two approaches can be found in Ref. [4]. We remark that in this paper exact analytical results are compared, and therefore, the estimation problem is not taken into account.

\subsection{Tavares-Lucena wavelet entropy}

Alternatively, Tavares and Lucena [2], following the basis entropy cost concept, have recently [24] defined another probability distribution:

$$
p_{j k}=\mathbb{E}\left|C_{j}(k)\right|^{2} / \mathscr{E}_{\text {tot }}^{(\mathrm{TL})} \quad \text { and } \quad p_{\phi}=\mathbb{E}|\langle\mathscr{S}, \phi\rangle|^{2} / \mathscr{E}_{\text {tot }}^{(\mathrm{TL})},
$$

where $\phi$ is the scaling function having the properties of a smoothing kernel (see Ref. [24] for details), and $\mathscr{E}_{\text {tot }}^{(\mathrm{TL})}=\sum_{j, k} \mathbb{E}\left|C_{j}(k)\right|^{2}+\mathbb{E}|\langle\mathscr{S}, \phi\rangle|^{2}$. Therefore, they propose the following entropy:

$$
S_{\mathrm{W}}^{(\mathrm{TL})}(N)=-\left(\sum_{j=-N+1}^{j=0} \sum_{k=0}^{2^{-j}-1} p_{j k} \log _{2} p_{j k}+p_{\phi} \log _{2} p_{\phi}\right) / S_{\max }^{(\mathrm{TL})},
$$

with $S_{\max }^{(\mathrm{TL})}=\log _{2}\left(2^{N}-1\right)$. As a matter of comparison we have normalized this expression and it will be referred as Tavares-Lucena Wavelet Entropy (TLWS).

It should be noted that in Eqs. (7), (8), and (10) the maximum resolution level $N$ is an experimental parameter. It appears explicitly as a direct consequence of sampling. Tavares and Lucena underlined this fact because it is not mentioned in previous approaches. 


\section{Theoretical results and comparison}

The aim of this paper is to study the performance of the wavelet entropy definitions previously given. So we analyze two well known stochastic processes, namely, the fBm and the fGn [28,29]. The energy per resolution level $j$ and sampled time $k$ has been already evaluated for the $\mathrm{fBm}[23,30,31]$. But it can be extended to fGnsee the Appendix. The final form reads

$$
\mathbb{E}\left|C_{j}^{\alpha}(k)\right|^{2}=2 c_{H}^{2} 2^{-j \alpha} \int_{0}^{\infty} v^{-\alpha}|\Psi(v)|^{2} \mathrm{~d} v
$$

where $-1<\alpha<3$ - by continuity we have added $\alpha=1$ but it does not belong to any existent process. It should be noted that the latter is independent of $k$. In the following we will use this power-law behavior with different ranges for $\alpha$, for the two stochastic processes under analysis, gathering both into a unified framework. According to its values, the coefficient $\alpha$ must be attached to one of the two mentioned processes.

In order to calculate the NTWS family, the relative wavelet energy for a finite data sample is obtained from Eqs. (5) and (11)

$$
p_{j}=2^{-(j+1)(\alpha-1)} \frac{1-2^{\alpha-1}}{1-2^{N(\alpha-1)}} .
$$

Similarly, the relative temporal average wavelet energy—see Eqs. (6) and (11) — gives

$$
\widetilde{p}_{j}=2^{-(j+1) \alpha} \frac{1-2^{\alpha}}{1-2^{N \alpha}} .
$$

Consequently, the normalized total wavelet entropies can be easily obtained from Eqs. (7) and (8)

$$
S_{\mathrm{W}}(N, \alpha)=\frac{(\alpha-1)}{\log _{2} N}\left[\frac{1}{1-2^{-(\alpha-1)}}-\frac{N}{1-2^{-N(\alpha-1)}}\right]-\frac{1}{\log _{2} N} \log _{2}\left[\frac{1-2^{(\alpha-1)}}{1-2^{N(\alpha-1)}}\right]
$$

and

$$
\widetilde{S}_{\mathrm{W}}(N, \alpha)=\frac{\alpha}{\log _{2} N}\left[\frac{1}{1-2^{-\alpha}}-\frac{N}{1-2^{-N \alpha}}\right]-\frac{1}{\log _{2} N} \log _{2}\left[\frac{1-2^{\alpha}}{1-2^{N \alpha}}\right] .
$$

For the Tavares and Lucena's approach similar steps should be followed. From the power-law behavior mentioned before a straightforward calculation yields

$$
p_{j k}=2^{-j \alpha} \frac{1-2^{\alpha+1}}{1-2^{N(\alpha+1)}} .
$$

Therefore, the TLWS is obtained replacing the above into Eq. (10),

$$
\begin{aligned}
S_{\mathrm{W}}^{(\mathrm{TL})}(N, \alpha)= & \frac{\alpha}{\log _{2}\left(2^{N}-1\right)}\left[\frac{1}{1-2^{-(\alpha+1)}}-\frac{N}{1-2^{-N(\alpha+1)}}\right] \\
& -\frac{1}{\log _{2}\left(2^{N}-1\right)} \log _{2}\left[\frac{1-2^{(\alpha+1)}}{1-2^{N(\alpha+1)}}\right] .
\end{aligned}
$$

The NTWS family and the TLWS, as a function of $\alpha$ and $N$, are depicted in Figs. 1-3. One point to emphasize from these graphs when $\alpha>0$ is that the NTWS's range of variation increases smoothly with $N$, improving detection; on the opposite, the TLWS's range decreases when $N$ increases. All entropies equally improve with $N$ on the $-1<\alpha<0$ branch. Moreover, for any $N$ the NTWS family covers almost all the available range between 0 and 1 , while the TLWS roughly covers a $25 \%$ of this range.

It is of common understanding that high entropy values are associated to a signal generated by a totally disordered random process, and low values to an ordered or partially ordered process. If the process is noisy, its signal wavelet decomposition is expected to have significant contributions to the total wavelet energy coming from all frequency bands. Moreover, one could expect that all the contributions being of the same order. Consequently, its relative energies will be almost equal at all resolution levels and acquire the entropy maximum value. While a nearly ordered process will have a relative energy contribution concentrated around 


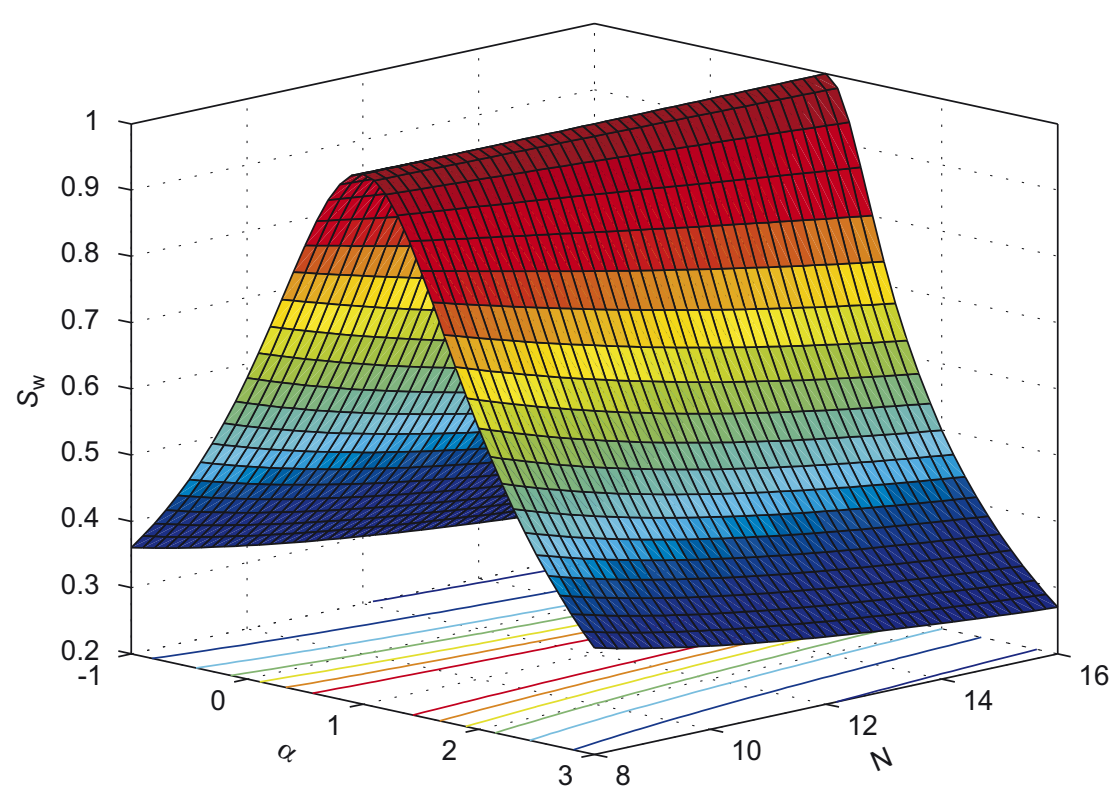

Fig. 1. NTWS entropy $S_{\mathrm{W}}$ as a function of $\alpha$ and $N$.

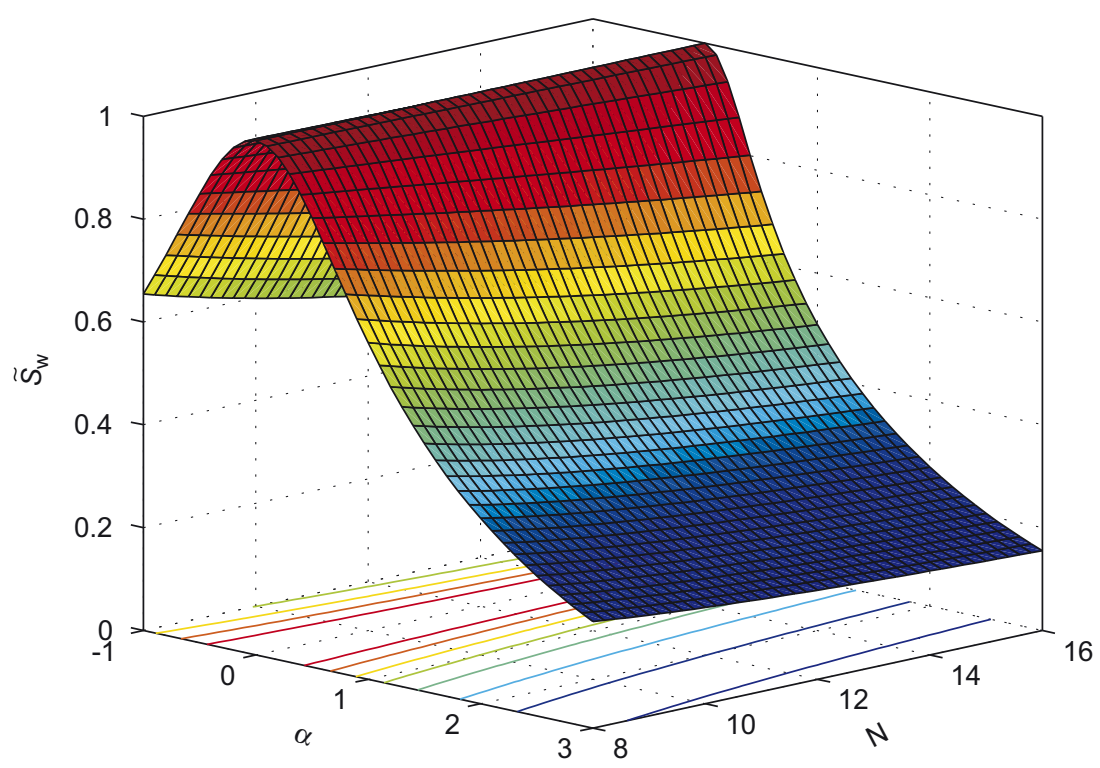

Fig. 2. NTWS entropy $\widetilde{S}_{\mathrm{W}}$ as a function of $\alpha$ and $N$.

some level $j$, thus its entropy will take a low value. The only entropy in concordance with this intuitive vision is $\widetilde{S}_{\mathrm{W}}$, depicted in Fig. 2.

In Fig. 4 we compare the two entropy formulations as functions of the $\alpha$-parameter when $N=12$. It is clear that the $\widetilde{S}_{\mathrm{W}}$ and $S_{\mathrm{W}}^{(\mathrm{TL})}$ entropies attain their maxima at $\alpha=0$ (white noise), and the $S_{\mathrm{W}}$ entropy reaches it when $\alpha \rightarrow 1$. There are two different regions to examine:

- $f B m, 1<\alpha<3$ : All the three quantifiers have their maximum at $\alpha \rightarrow 1$, and monotonically decrease to find their minimum in a near regular process, $\alpha \rightarrow 3$. The range of variation of the TLWS is $\Delta S_{\mathrm{W}}^{(\mathrm{TL})}=0.038$, and 


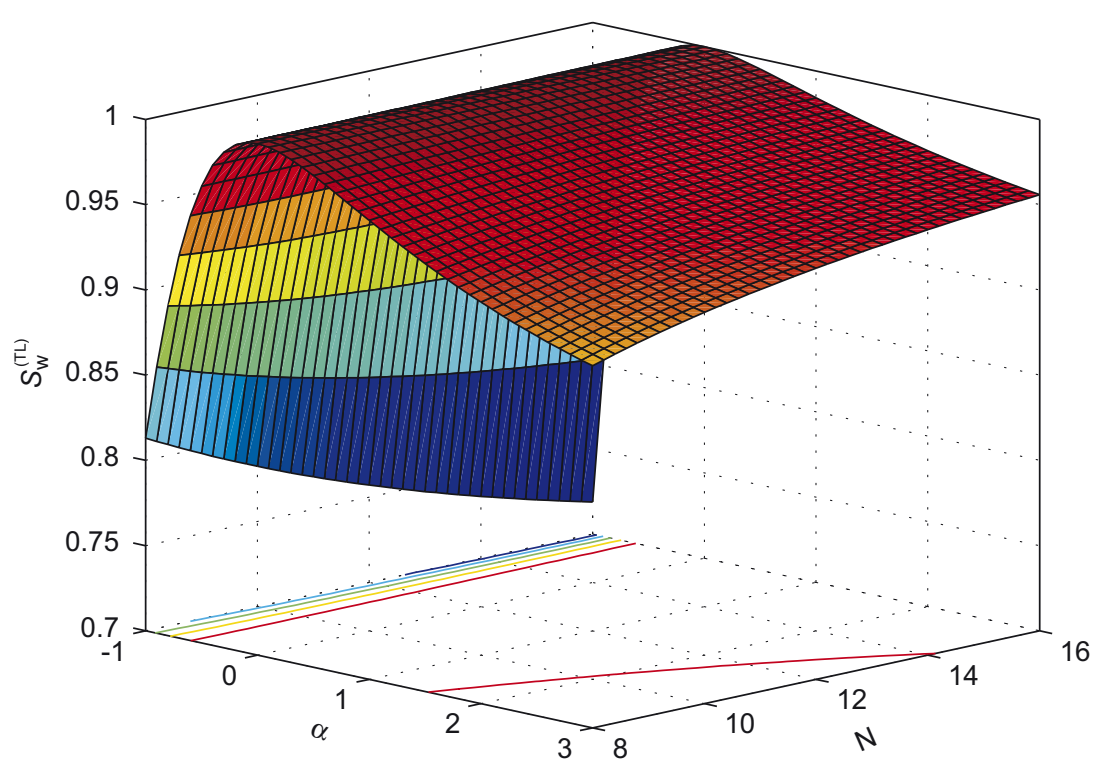

Fig. 3. TLWS entropy $S_{\mathrm{W}}^{(\mathrm{TL})}$ as a function of $\alpha$ and $N$.

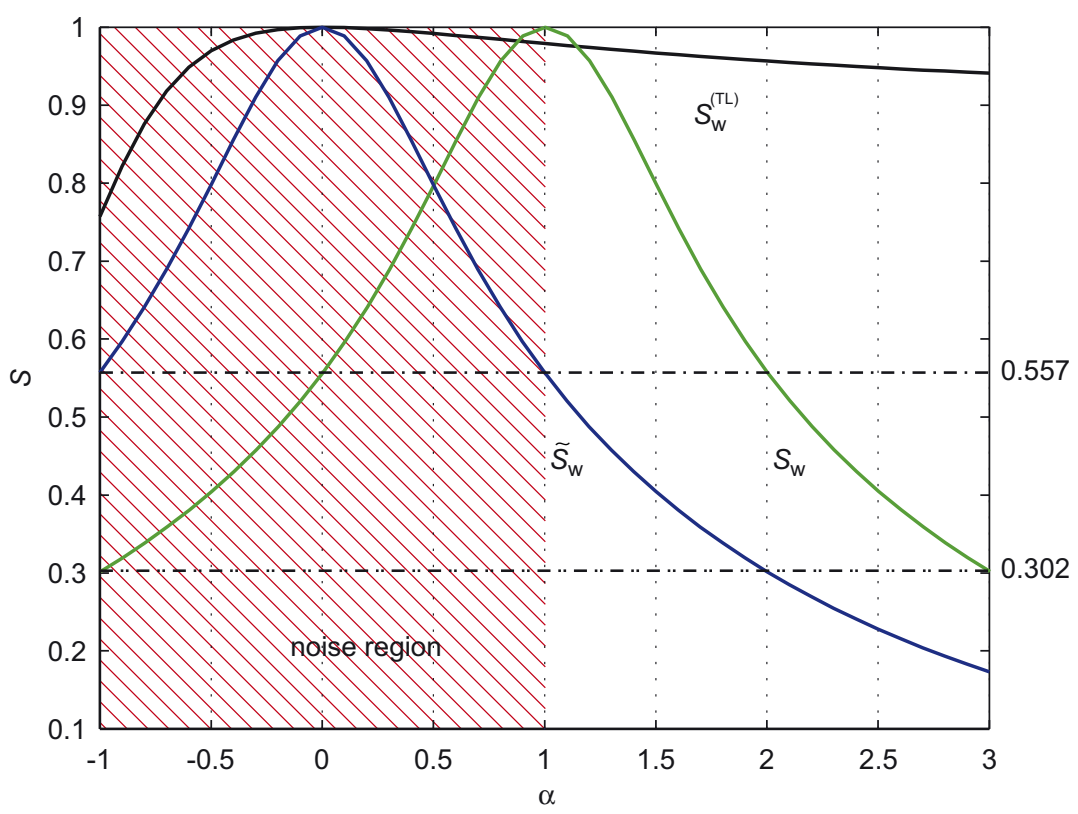

Fig. 4. NTWS and TLWS as functions of $\alpha$ with $N=12$.

the range of variation of the NTWS family is $\Delta \widetilde{S}_{\mathrm{W}}=0.384$ and $\Delta S_{\mathrm{W}}=0.698$. Clearly, due to the small range of variation, the TLWS is unfit to differentiate between the short- and long-memory fBm family members, $1<\alpha<2$ and $2<\alpha<3$, respectively. The NTWS family seems to be the best tool for this differentiation, and the $S_{\mathrm{W}}$ has the best performance in this interval.

- $f G n,-1<\alpha<1$ : The TLWS seems inadequate to describe this range-note that $S_{\mathrm{W}}^{(\mathrm{TL})}(12,-1)<S_{\mathrm{W}}^{(\mathrm{TL})}(12,3)$. The $S_{\mathrm{W}}$ is best suited to describe these noises, since it is monotonically decreasing and presents a range of variation $\Delta S_{\mathrm{W}}=0.698$. While the $\widetilde{S}_{\mathrm{W}}$ confuses between noises coming from short- or long-memory processes, $-1<\alpha<0$ and $0<\alpha<1$, respectively. It has its maximum at $\alpha=0$ (white noise). 


\section{Conclusions}

We have introduced exact theoretical expressions for the wavelet entropies associated to fGn, $-1<\alpha<1$. In particular, the range $-1<\alpha<0$, to our knowledge, has never been studied.

We have shown that, at least to characterize fBm's and fGn's processes, the NTWS family seems to be a better quantifier than TLWS. The $\widetilde{S}_{\mathrm{W}}$ fulfills all the requirements for a correct description of the overall $\alpha$-range: has its maximum at the white noise, differentiates between noises and processes, and has the maximum range of variation, $\Delta \widetilde{S}_{\mathrm{W}}=0.827$. Nevertheless, the $S_{\mathrm{W}}$ is best suited to discern between different fBm processes. Finally, in the $\alpha>0$ case, an inverse dependence on $N$ is observed: the NTWS family increases its performance as $N$ increases and the TLWS improves its performance as $N$ decreases. Although the NTWS family always improves with $N$ for any $\alpha$ value.

The procedure outlined in Section 2.1 can be followed to build new probability distributions associated to the wavelet resolution levels. For example, if instead of the number of coefficients per resolution level, $N_{j}$, as weight factor in Eq. (4), we use a power of it, $N_{j}^{\beta}$, then the $\alpha$-parameter where the NTWS attains its maximum changes according to this weight. So, the probability distribution could be modified depending of the requirements of the physical problem under study. In particular, the $\widetilde{S}_{\mathrm{W}}$ agrees with the popular conception of maximum entropy for the white noise $(\alpha=0)$.

It should be stressed that it is not the intention of this paper to compare the behavior of the aforementioned quantifiers with other techniques for the study of complex signals [32-34]. That task will be the challenge of future works.

\section{Acknowledgments}

This work was partially supported by Consejo Nacional de Investigaciones Científicas y Técnicas (PIP 5687/ 05, CONICET, Argentina), Comisión Nacional de Investigación Científica y Tecnológica (CONICYT, FONDECYT Project No. 11060512, Chile), and Pontificia Universidad Católica de Valparaíso (PUCV, Project No. 123.786/2006, Chile). D.G.P. and O.A.R. are very grateful to Prof. Dr. Javier Martínez-Mardones for his kind hospitality at Instituto de Física, Pontificia Universidad Católica de Valparaíso, Chile, where part of this work was done.

\section{Appendix}

Given any Wiener space, generalized random variables $X(\omega)$, with $\omega$ one element of the statistic ensemble, can be defined through the formal sum, called chaos expansion, [35]

$$
X(\omega)=\sum_{\gamma} c_{\gamma} \mathscr{H}_{\gamma}(\omega) \quad \text { with } c_{\gamma}^{2}=\mathbb{E}\left[X \mathscr{H}_{\gamma}\right] / \gamma !
$$

Here $\gamma !=\gamma_{1} ! \gamma_{2} ! \cdots \gamma_{n} !$ is the factorial of the finite non-negative integer multi-index $\gamma$, while $\mathscr{H}_{\gamma}(\omega)=$ $\prod_{i=1}^{n} H_{\gamma_{i}}\left(\left\langle\xi_{i}, \omega\right\rangle\right)$ represents the stochastic component of the process, and it is build up through the Itô integrals $\left\langle\xi_{i}, \omega\right\rangle$ of Hermite functions:

$$
\xi_{n}(x)=\frac{\mathrm{e}^{-x^{2} / 2} H_{n-1}(x)}{\sqrt{2^{n-1}(n-1) ! \pi^{1 / 2}}}, \quad n=1,2, \ldots,
$$

with $H_{n}$ the Hermite polynomials. This is an orthogonal basis, and thus fulfills

$$
\sum_{n=1}^{\infty} \xi_{n}(x) \xi_{n}\left(x^{\prime}\right)=\delta\left(x-x^{\prime}\right) .
$$

In particular, Gaussian processes, with zero mean, attain the simplest chaos expansion, i.e.,

$$
X(\omega)=\sum_{n=1}^{\infty} c_{n} \mathscr{H}_{\varepsilon_{n}}(\omega)
$$


where $\varepsilon_{n}=(0,0, \ldots, 0,1,0, \ldots)$ with 1 on the $n$th entry, and 0 otherwise, so $\mathscr{H}_{\varepsilon_{n}}(\omega)=\left\langle\xi_{n}, \omega\right\rangle$. Furthermore, the covariance of two Gaussian processes, $X(\omega)=\sum_{n=1}^{\infty} c_{n} \mathscr{H}_{\varepsilon_{n}}(\omega)$ and $Y(\omega)=\sum_{n=1}^{\infty} d_{n} \mathscr{H}_{\varepsilon_{n}}(\omega)$, has a simple expression through the expansion of their coefficients [35, p. 43]:

$$
\mathbb{E} X Y=\sum_{n=1}^{\infty} c_{n} d_{n}
$$

There is a particular Wiener space for fractional Brownian processes where a stochastic calculus can be developed for the complete range of the Hurst parameter. Elliott and van der Hoek [36] were the first to introduce it, and we will use it through this appendix. Processes within this Wiener space are built around the self-adjoint operator $M_{H}$ defined as

$$
\widehat{M_{H}} \phi(v)=c_{H}|v|^{1 / 2-H} \widehat{\phi}(v),
$$

where the hat $\widehat{ }$ stands for the Fourier transform, $c_{H}^{2}=\Gamma(2 H+1) \sin (\pi H)$, and $\phi$ is any function such that $\widehat{M_{H}} \phi \in L^{2}(\mathbb{R})$. In particular, the chaos expansion for fBm results

$$
B^{H}(t, \omega)=\sum_{n=1}^{\infty}\left(M_{H} \mathbb{1}_{[0, t]}, \xi_{n}\right) \mathscr{H}_{\varepsilon_{n}}(\omega),
$$

see Ref. [36] for further details. Since $\omega$ is fixed, whenever its presence is unnecessary it will be omitted. Since the operator $M_{H}$ is self-adjoint,

$$
\left(M_{H} \mathbb{1}_{[0, t]}, \xi_{n}\right)=\left(\mathbb{1}_{[0, t]}, M_{H} \xi_{n}\right)=\int_{0}^{t} \mathrm{~d} s M_{H} \xi_{n}(s) .
$$

Henceforth, the fractional white noise has the expansion

$$
\frac{\mathrm{d}}{\mathrm{d} t} B^{H}(t)=\sum_{n=1}^{\infty} M_{H} \xi_{n}(t) \mathscr{H}_{\varepsilon_{n}}(\omega)=W^{H}(t) .
$$

Following the methodology employed in Pérez et al. [23] to find the chaos expansion of the wavelet coefficients for the fBm, let us take as the signal the noise $s(t)=W^{H}(t, \omega)$. Then, given the orthonormal wavelet basis $\left\{2^{j / 2} \psi\left(2^{j} \cdot-k\right)\right\}_{j, k \in \mathbb{Z}}=\left\{\psi_{j, k}\right\}_{j, k \in \mathbb{Z}}$, we obtain the wavelet coefficient expansion:

$$
C_{j}^{W^{H}}(k)=\left(W^{H}, \psi_{j, k}\right)=\sum_{n=1}^{\infty}\left(M_{H} \xi_{n}, \psi_{j, k}\right) \mathscr{H}_{\varepsilon_{n}}(\omega) .
$$

Each one of these coefficients is also a Gaussian process [35]. Since we are interested in evaluating their covariance, because of Eq. (22), we just need to work with the individual coefficients

$$
d_{n}^{H}(j, k)=\left(M_{H} \xi_{n}, \psi_{j, k}\right)=c_{H} \int_{\mathbb{R}}|v|^{1 / 2-H} \widehat{\xi}_{n}^{*}(v) \widehat{\psi}_{j, k}(v) \mathrm{d} v .
$$

Since, the Fourier transforms of the Hermite functions and the wavelet are $\widehat{\xi}_{n}^{*}(v)=\mathrm{i}^{n-1} \xi_{n}(v)$ and $\widehat{\psi}_{j, k}(v)=2^{-j} \exp \left(-\mathrm{i} 2^{-j} k v\right) \widehat{\psi}\left(2^{-j} v\right)$, respectively. The evaluation of the coefficients $d_{n}^{H}(j, k)$ is straightforward from their definition:

$$
d_{n}^{H}(j, k)=c_{H} i^{n-1} 2^{-(H-1 / 2) j} \int_{\mathbb{R}}|v|^{1 / 2-H} \Psi(v) 2^{j / 2} \xi_{n}\left(2^{j} v\right) \mathrm{e}^{-\mathrm{i} k v} \mathrm{~d} v,
$$

where $\Psi(v)=\widehat{\psi}(v)$. 
Under the same procedure used in Ref. [23] the second moment of any wavelet coefficient can be calculated using Eq. (22), as follows:

$$
\begin{aligned}
\mathbb{E}\left|C_{j}^{W^{H}}(k)\right|^{2} & =\sum_{n=1}^{\infty} d_{n}^{H}(j, k)^{*} d_{n}^{H}\left(j, k^{\prime}\right) \\
& =c_{H}^{2} 2^{-j(2 H-1)} \int_{\mathbb{R}^{2}}|v|^{-(H-1 / 2)}\left|v^{\prime}\right|^{-(H-1 / 2)} \Psi(v) \Psi^{*}\left(v^{\prime}\right) \mathrm{e}^{-\mathrm{i} k\left(v-v^{\prime}\right)} 2^{j} \sum_{n=1}^{\infty} \xi_{n}\left(2^{j} v\right) \xi_{n}\left(2^{j} v^{\prime}\right) \mathrm{d} v \mathrm{~d} v^{\prime} \\
& =2 \Gamma(2 H+1) \sin (\pi H) 2^{-j(2 H-1)} \int_{0}^{\infty} v^{-(2 H-1)}|\Psi(v)|^{2} \mathrm{~d} v,
\end{aligned}
$$

for the last step we used $\sum_{n=1}^{\infty} \xi_{n}\left(2^{j} v\right) \xi_{n}\left(2^{j} v^{\prime}\right)=2^{-j} \delta\left(v-v^{\prime}\right)$, from property (20), and the parity of $\Psi$-also, it should decay fast enough for the integral to converge.

In the case of the fractional Gaussian noises $\alpha=2 H-1$, as opposite to the fractional Brownian motion where $\alpha=2 H+1$. For the latter we have previously reported [23] that

$$
\mathbb{E}\left|C_{j}^{B^{H}}(k)\right|^{2}=2 \Gamma(2 H+1) \sin (\pi H) 2^{-j(2 H+1)} \int_{0}^{\infty} v^{-(2 H+1)}|\Psi(v)|^{2} \mathrm{~d} v
$$

for any mother wavelet satisfying $\int_{\mathbb{R}} \psi=0$. Therefore, these two expressions, Eqs. (29) and (30), can be combined in one written in terms of the power $\alpha$ :

$$
\mathbb{E}\left|C_{j}^{\alpha}(k)\right|^{2}=2 c_{H}^{2} 2^{-j \alpha} \int_{0}^{\infty} v^{-\alpha}|\Psi(v)|^{2} \mathrm{~d} v
$$

where $-1<\alpha<1$ or $1<\alpha<3$, and $c_{H}$ is calculated from the value of $\alpha$.

\section{References}

[1] I. Daubechies, Ten Lectures on Wavelets, SIAM, Philadelphia, 1992.

[2] S. Mallat, A Wavelet Tour of Signal Processing, second ed., Academic Press, New York, 1999.

[3] S. Blanco, A. Figliola, R.Q. Quiroga, O.A. Rosso, E. Serrano, Time-frequency analysis of electroencephalogram series, III. Wavelet packets and information cost function, Phys. Rev. E 57 (1998) 932-940.

[4] O.A. Rosso, S. Blanco, J. Yordanova, V. Kolev, A. Figliola, M. Schürmann, E. Başar, Wavelet entropy: a new tool for analysis of short duration brain electrical signals, J. Neurosci. Method 105 (2001) 65-75.

[5] O.A. Rosso, M.L. Mairal, Characterization of time dynamical evolution of electroencephalographic epileptic records, Physica A 312 (3-4) (2002) 469-504.

[6] O.A. Rosso, M.T. Martín, A. Plastino, Brain electrical activity analysis using wavelet based informational tools, Physica A 313 (2002) 587-608.

[7] H. Hasegawa, Stochastic resonance of ensemble neurons for transient spike trains: wavelet analysis, Phys. Rev. E 66 (2) (2002) 021902.

[8] O.A. Rosso, S. Blanco, A. Rabinowicz, Wavelet analysis of generalized tonic-clonic epileptic seizures, Signal Process. 86 (2003) 1275-1289.

[9] H.A. Al-Nashash, J.S. Paul, W.C. Ziai, D.F. Hanley, N.V. Thakor, Wavelet entropy for subband segmentation of EEG during injury and recovery, Ann. Biomed. Eng. 31 (2003) 653-658.

[10] O.A. Rosso, W. Hyslop, R. Gerlach, R.L.L. Smith, J.A.P. Rostas, M. Hunter, Quantitative EEG analysis of the maturational changes associated with childhood absence epilepsy, Physica A 356 (1) (2005) 184-189.

[11] H.A. Al-Nashash, N.V. Thakor, Monitoring of global cerebral ischemia using wavelet entropy rate of change, IEEE Trans. Biomed. Eng. 52 (12) (2005) 2119-2122.

[12] H.C. Shin, S. Tong, S. Yamashita, X. Jia, R.G. Geocadin, N.V. Thakor, Quantitative EEG and effect of hypothermia on brain recovery after cardiac arrest, IEEE Trans. Biomed. Eng. 53 (6) (2006) 1016-1023.

[13] A.W.L. Chiu, S.S. Jahromi, H. Khosravani, P.L. Carlen, B.L. Bardakjian, The effects of high-frequency oscillations in hippocampal electrical activities on the classification of epileptiform events using artificial neural networks, J. Neural Eng. 3 (1) (2006) 9-20.

[14] O.A. Rosso, M.T. Martin, A. Figliola, K. Keller, A. Plastino, EEG analysis using wavelet-based information tools, J. Neurosci. Method 153 (2) (2006) 163-182.

[15] S. Sello, Wavelet entropy as a measure of solar cycle complexity, Astron. Astrophys. 363 (2000) 311-315.

[16] S. Sello, Wavelet entropy and the multi-peaked structure of solar cycle maximum, New Astron. 8 (2003) $105-117$.

[17] A.M. Korol, R.J. Rasia, O.A. Rosso, Alterations of thalassemic erythrocytes detected by wavelet entropy, Physica A 375 (1) (2007) 257-264. 
[18] L. Zunino, D.G. Pérez, M. Garavaglia, O.A. Rosso, Characterization of laser propagation through turbulent media by quantifiers based on the wavelet transform, Fractals 12 (2) (2004) 223-233.

[19] L. Zunino, D.G. Pérez, M. Garavaglia, O.A. Rosso, Characterization of laser propagation through turbulent media by quantifiers based on the wavelet transform: dynamic study, Physica A 364 (2006) 79-86.

[20] I. Passoni, A. Dai Pra, H. Rabal, M. Trivi, R. Arizaga, Dynamic speckle processing using wavelets based entropy, Opt. Commun. 246 (1-3) (2005) 219-228.

[21] C.M. González, H.A. Larrondo, O.A. Rosso, Statistical complexity measure of pseudorandom bit generators, Physica A 354 (2005) 281-300.

[22] A.M. Kowalski, M.T. Martín, A. Plastino, A.N. Proto, O.A. Rosso, Wavelet statistical complexity analysis of the classical limit, Phys. Lett. A 311 (2003) 180-191.

[23] D.G. Pérez, L. Zunino, M. Garavaglia, O.A. Rosso, Wavelet entropy and fractional Brownian motion time series, Physica A 365 (2) (2006) 282-288.

[24] D.M. Tavares, L.S. Lucena, Entropy analysis of stochastic processes at finite resolution, Physica A 357 (1) (2005) $71-78$.

[25] A. Carbone, G. Castelli, H.E. Stanley, Time-dependent Hurst exponent in financial time series, Physica A 344 (1-2) (2004) 267-271.

[26] P.S. Addison, A.S. Ndumu, Engineering applications of fractional Brownian motion: self-affine and self-similar random processes, Fractals 7 (2) (1999) 151-157.

[27] C.E. Shannon, A mathematical theory of communications, Bell Syst. Technol. J. 27 (1948) 379-423 and 623-656.

[28] B.B. Mandelbrot, J.W. Van Ness, Fractional Brownian motions, fractional noises and applications, SIAM Rev. 4 (1968) $422-437$.

[29] G. Samorodnitsky, M.S. Taqqu, Stable non-Gaussian random processes: stochastic models with infinite variance, Chapman \& Hall/ CRC, London, UK, 1994.

[30] P. Flandrin, Wavelet analysis and synthesis of fractional Brownian motion, IEEE Trans. Inform. Theory IT-38 (2) (1992) 910-917.

[31] P. Abry, P. Flandrin, M.S. Taqqu, D. Veitch, Wavelets for the analysis, estimation, and synthesis of scaling data, in: K. Park, W. Willinger (Eds.), Self-similar Network Traffic and Performance Evaluation, Wiley, New York, 2000.

[32] G. Boffetta, M. Cencini, M. Falcioni, A. Vulpiani, Predictability: a way to characterize complexity, Phys. Reports 356 (2002) 367-474.

[33] K. Urbanowicz, J.A. Holyst, Noise-level estimation of time series using coarse-grained entropy, Phys. Rev. E 67 (2003) 046218.

[34] M. Anghel, On the effective dimension and dynamic complexity of earthquake faults, Chaos, Solitons \& Fractals 19 (2) (2004) 399-420.

[35] H. Holden, B. Øksendal, J. Ubøe, T. Zhang, Stochastic Partial Differential Equations: a Modeling, White Noise Functional Approach, Probability and Its Applications, Birkhäuser, Boston, 1996.

[36] R.J. Elliott, J. van der Hoek, A general fractional white noise theory and applications to finance, Math. Finance 13 (2003) 301-330. 